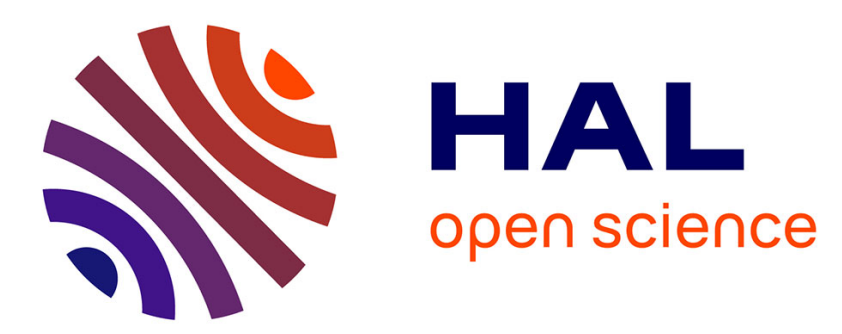

\title{
Asymptotic normality of sample covariance matrix for mixed spectra time series: Application to sinusoidal frequencies estimation \\ Jean-Pierre Delmas
}

\section{- To cite this version:}

Jean-Pierre Delmas. Asymptotic normality of sample covariance matrix for mixed spectra time series: Application to sinusoidal frequencies estimation. IEEE Transactions on Information Theory, 2001. hal-03399917

\section{HAL Id: hal-03399917 https://hal.science/hal-03399917}

Submitted on 24 Oct 2021

HAL is a multi-disciplinary open access archive for the deposit and dissemination of scientific research documents, whether they are published or not. The documents may come from teaching and research institutions in France or abroad, or from public or private research centers.
L'archive ouverte pluridisciplinaire HAL, est destinée au dépôt et à la diffusion de documents scientifiques de niveau recherche, publiés ou non, émanant des établissements d'enseignement et de recherche français ou étrangers, des laboratoires publics ou privés. 


\title{
Asymptotic normality of sample covariance matrix for
}

\author{
mixed spectra time series:
}

\section{Application to sinusoidal frequencies estimation}

\author{
Jean-Pierre Delmas
}

\begin{abstract}
This correspondence addresses the asymptotic normal distribution of the sample mean and the sample covariance matrix of mixed spectra time series containing a sum of sinusoids and a moving average process. Two central limit theorems are proved. As an application of this result, the asymptotic normal distribution of any sinusoidal frequencies estimator of such time series based on second-order statistics is deduced.
\end{abstract}

Index terms: central limit theorem, sample covariance matrix, mixed spectra time series, covariancebased sinusoidal frequencies estimation.

Final version of correspondence IT-CLN 99-170

Département Signal et Image, Institut National des Télécommunications, 9 rue Charles Fourier, 91011 Evry Cedex, FRANCE. Fax: +33-1-60 7644 33, e-mail: jean-pierre.delmas@int-evry.fr. 


\section{Introduction}

There is considerable literature (e.g. [1]-[4]) concerning the asymptotic Gaussian distribution of sample mean and sample covariance matrix of real-valued stationary processes $x_{t}$. Several situations have been considered, among them $x_{t}$ is a generalized linear process, satisfies mixing conditions or is Gaussian with a power spectral density. However, few contribution have been devoted to the asymptotic distributions of the sample covariance matrix associated with mixed spectra time series. Subsequent to the revision of this manuscript, the reference [5] which was brought to our attention, tackled this problem but with quite advanced statistical tools such as martingale theory.

We will be concerned with real or complex ${ }^{1}$ valued processes of the type

$$
x_{t}=m+\sum_{k=1}^{K} a_{k} \cos \left(2 \pi f_{k} t+\phi_{k}\right)+v_{t} \quad \text { or } \quad x_{t}=m+\sum_{k=1}^{K} a_{k} e^{i \phi_{k}} e^{i 2 \pi f_{k} t}+v_{t}
$$

with

$$
v_{t}=\sum_{q=0}^{Q} b_{q} u_{t-q} .
$$

Throughout this paper, $\left(u_{t}\right)_{t=1, \ldots, n}$ is a sequence of zero-mean i.i.d. random variables where $\mathrm{E}\left|u_{t}^{4}\right|<\infty$, with

$c_{u} \stackrel{\text { def }}{=} \mathrm{E}\left(u_{t}^{2}\right), \kappa_{u} \stackrel{\text { def }}{=} \operatorname{Cum}\left(u_{t}, u_{t}, u_{t}, u_{t}\right)$ and $c_{u} \stackrel{\text { def }}{=} \mathrm{E}\left|u_{t}^{2}\right|, c_{u}^{\prime} \stackrel{\text { def }}{=} \mathrm{E}\left(u_{t}^{2}\right), \kappa_{u} \stackrel{\text { def }}{=} \operatorname{Cum}\left(u_{t}, u_{t}^{*}, u_{t}, u_{t}^{*}\right)$, respectively in the real and the possibly noncircular ${ }^{2}$ complex case. $\left(a_{k}\right)_{k=1, \ldots, K}$ and $\left(b_{q}\right)_{q=0, \ldots, Q}$ are unknown fixed real or complex numbers respectively. $m$ is an unknown fixed number and $f_{k}$ are unknown fixed distinct real numbers in $] 0,1 / 2[$ for real valued processes [resp. in ] $-1 / 2,+1 / 2$ [ for complex valued processes]. For the phases $\phi_{k}$, the model (1.1) can be interpreted in two different ways, leading to different statistical descriptions.

1. We can assume that $\phi_{k}$ are random variables uniformly distributed on $[0,2 \pi]$ and that $\left(\phi_{k}\right)_{k=1, \ldots, K}$ and $u_{t}$ are mutually independent. In this case $x_{t}$ is a wide-sense stationary process.

2. We can assume that $\phi_{k}$ are nonrandom unknown parameters ${ }^{3}$ and so $x_{t}$ is a not a wide-sense stationary process.

We are interested in the asymptotic distribution of the sample mean $m_{n} \stackrel{\text { def }}{=} \frac{1}{n} \sum_{t=1}^{n} x_{t}$ and of the sample covariance matrix $\mathbf{R}_{n} \stackrel{\text { def }}{=} \frac{1}{n} \sum_{t=1}^{n}\left(\mathbf{x}_{t}-\mathbf{m}_{n}\right)\left(\mathbf{x}_{t}-\mathbf{m}_{n}\right)^{T}$ in the real case [resp., $\mathbf{R}_{n} \stackrel{\text { def }}{=} \frac{1}{n} \sum_{t=1}^{n}\left(\mathbf{x}_{t}-\mathbf{m}_{n}\right)\left(\mathbf{x}_{t}-\mathbf{m}_{n}\right)^{H}$ in the complex case], where $\mathbf{x}_{t} \stackrel{\text { def }}{=}\left(x_{t}, x_{t-1}, \ldots, x_{t-p+1}\right)^{T}$ and $\mathbf{m}_{n} \stackrel{\text { def }}{=}\left(m_{n}, \ldots, m_{n}\right)^{T}$. The asymptotic normality of the sample mean $m_{n}$ and sample covariance matrix $\mathbf{R}_{n}$ is proved in Section 2. As an application of this result, the

\footnotetext{
${ }^{1}$ Complex processes appear as complex envelope of bandpass real processes.

${ }^{2}$ Here, circular refers to second-order circular (see e.g. [6] which is sometimes called "proper" (see [7]).

${ }^{3}$ In this model $f_{k} \neq 0$, otherwise $m$ would be a special case of a sinusoid.
} 
asymptotic normal distribution of any sinusoidal frequencies estimator of such time series, based on second-order statistics, is derived in Section 3.

\section{Central limit theorems}

For the convenience of the reader, the definition of the complex Gaussian distribution is recalled. A complex random $p \times 1$ vector $\mathbf{y}$ has a zero-mean complex Gaussian distribution specified by $p \times p$ positive definite matrix $\boldsymbol{\Sigma}_{1}$ and $p \times p$ symmetric matrix $\boldsymbol{\Sigma}_{2}$ and denoted $\mathcal{N}\left(\mathbf{0} ; \boldsymbol{\Sigma}_{1}, \boldsymbol{\Sigma}_{2}\right){ }^{4}$ if the $2 p$-joint distribution of the real and imaginary part of $\mathbf{y}$ is $2 p$-zero-mean real Gaussian, i.e. for any complex $p \times 1$ vector $\mathbf{w}$ : the real scalar $\mathbf{w}^{H} \mathbf{y}+\left(\mathbf{w}^{H} \mathbf{y}\right)^{H}$ has a zero-mean real Gaussian distribution with variance

$$
2 \mathbf{w}^{H} \boldsymbol{\Sigma}_{1} \mathbf{w}+\mathbf{w}^{H} \boldsymbol{\Sigma}_{2} \mathbf{w}^{*}+\mathbf{w}^{T} \boldsymbol{\Sigma}_{2}^{*} \mathbf{w}
$$

where $\mathrm{E}\left(\mathbf{y} \mathbf{y}^{H}\right)=\boldsymbol{\Sigma}_{1}$ and $\mathrm{E}\left(\mathbf{y} \mathbf{y}^{T}\right)=\boldsymbol{\Sigma}_{2}$.

Considering the sample mean, the following theorem is proved in the Appendix.

Theorem $1 \sqrt{n}\left(m_{n}-m\right)$ converges in distribution to the zero-mean real [resp., complex] Gaussian distribution of variance $c_{m}\left[\right.$ resp., $c_{m}, c_{m}^{\prime}$ ] in the real case [resp., in the complex case] irrespective of the phase model.

$$
\sqrt{n}\left(m_{n}-m\right) \stackrel{\mathcal{L}}{\rightarrow} \mathcal{N}\left(0, c_{m}\right) \quad\left[\text { resp., } \mathcal{N}\left(0 ; c_{m}, c_{m}^{\prime}\right)\right] .
$$

Furthermore

$$
\lim _{n \rightarrow \infty} \mathrm{E}\left(m_{n}\right)=m \quad \text { and } \quad \lim _{n \rightarrow \infty} n \operatorname{Var}\left(m_{n}\right)=c_{m}
$$

where $c_{m}$ and $c_{m}^{\prime}$ are defined as:

$$
c_{m}=c_{u}\left|\sum_{q=0}^{Q} b_{q}\right|^{2} \quad \text { and } \quad c_{m}^{\prime}=c_{u}^{\prime}\left(\sum_{q=0}^{Q} b_{q}\right)^{2} .
$$

Then, considering the sample covariance matrix, if $\mathbf{R}$ denotes respectively $\mathrm{E}\left[\left(\mathbf{x}_{t}-\mathbf{m}\right)\left(\mathbf{x}_{t}-\mathbf{m}\right)^{T}\right]$ and $\mathrm{E}\left[\left(\mathbf{x}_{t}-\right.\right.$ $\left.\mathbf{m})\left(\mathbf{x}_{t}-\mathbf{m}\right)^{H}\right]$ in the real and the complex case of the first statistical model with $\mathbf{m} \stackrel{\text { def }}{=}(m, \ldots, m)^{T}$, the following theorem is proved in the Appendix.

\footnotetext{
${ }^{4}$ This notation was introduced by [6]. The matrix $\boldsymbol{\Sigma}_{2}$ is respectively called relation matrix by [6] and pseudo-covariance matrix by [7].
} 
Theorem $2 \sqrt{n}\left(\operatorname{Vec}\left(\mathbf{R}_{n}\right)-\operatorname{Vec}(\mathbf{R})\right)^{5}$ converges in distribution to the zero-mean real [resp., complex] Gaussian distribution of covariance $\mathbf{C}_{R}$ [resp., $\mathbf{C}_{R}, \mathbf{C}_{R} \mathbf{K}$ ] in the real case [resp., in the complex case], irrespective of the phase model.

$$
\sqrt{n}\left(\operatorname{Vec}\left(\mathbf{R}_{n}\right)-\operatorname{Vec}(\mathbf{R})\right) \stackrel{\mathcal{L}}{\rightarrow} \mathcal{N}\left(\mathbf{0}, \mathbf{C}_{R}\right) \quad\left[\text { resp., } \mathcal{N}\left(\mathbf{0}, \mathbf{C}_{R}, \mathbf{C}_{R} \mathbf{K}\right)\right]
$$

Furthermore

$$
\lim _{n \rightarrow \infty} \mathrm{E}\left(\mathbf{R}_{n}\right)=\mathbf{R} \quad \text { and } \quad \lim _{n \rightarrow \infty} n \operatorname{Cov}\left(\operatorname{Vec}\left(\mathbf{R}_{n}\right)\right)=\mathbf{C}_{R}
$$

where $\mathbf{C}_{R}$ is defined respectively in the real and the complex cases as:

$$
\begin{aligned}
\mathbf{C}_{R}=\int_{-1 / 2}^{+1 / 2} & S_{v}^{2}(f)\left[\mathbf{e}(f) \mathbf{e}^{H}(f) \otimes_{c} \mathbf{e}(f) \mathbf{e}^{H}(f)+\mathbf{e}(f) \mathbf{e}^{T}(f) \otimes_{c} \mathbf{e}(f) \mathbf{e}^{T}(f)\right] d f+\kappa_{u} \operatorname{Vec}\left(\mathbf{B B}^{T}\right) \operatorname{Vec}^{T}\left(\mathbf{B B}^{T}\right) \\
& +\frac{1}{2} \sum_{k=1}^{K} a_{k}^{2} S_{v}\left(f_{k}\right)\left[\mathbf{e}\left(f_{k}\right) \mathbf{e}^{H}\left(f_{k}\right) \otimes_{c} \mathbf{e}\left(f_{k}\right) \mathbf{e}^{H}\left(f_{k}\right)+\mathbf{e}\left(-f_{k}\right) \mathbf{e}^{H}\left(-f_{k}\right) \otimes_{c} \mathbf{e}\left(-f_{k}\right) \mathbf{e}^{H}\left(-f_{k}\right)\right] \\
& +\frac{1}{2} \sum_{k=1}^{K} a_{k}^{2} S_{v}\left(f_{k}\right)\left[\mathbf{e}\left(f_{k}\right) \mathbf{e}^{H}\left(-f_{k}\right) \otimes_{c} \mathbf{e}\left(f_{k}\right) \mathbf{e}^{H}\left(-f_{k}\right)+\mathbf{e}\left(-f_{k}\right) \mathbf{e}^{H}\left(f_{k}\right) \otimes_{c} \mathbf{e}\left(-f_{k}\right) \mathbf{e}^{H}\left(f_{k}\right)\right] \\
\mathbf{C}_{R}=\int_{-1 / 2}^{+1 / 2} S_{v}^{2}(f)\left[\mathbf{e}(f) \mathbf{e}^{H}(f) \otimes_{c} \mathbf{e}(f) \mathbf{e}^{H}(f)\right] d f+\int_{-1 / 2}^{+1 / 2}{S^{\prime}}_{v}^{2}(f)\left[\mathbf{e}(f) \mathbf{e}^{T}(f) \otimes_{c} \mathbf{e}(f) \mathbf{e}^{T}(f)\right] d f & \\
& +2 \sum_{k=1}^{K} a_{k}^{2} S_{v}\left(f_{k}\right)\left[\mathbf{e}\left(f_{k}\right) \mathbf{e}^{H}\left(f_{k}\right) \otimes_{c} \mathbf{e}\left(f_{k}\right) \mathbf{e}^{H}\left(f_{k}\right)\right]+\kappa_{u} \operatorname{Vec}\left(\mathbf{B B}^{H}\right) \operatorname{Vec}^{H}\left(\mathbf{B B}^{H}\right)
\end{aligned}
$$

with $\mathbf{e}(f) \stackrel{\text { def }}{=}\left(1, e^{i 2 \pi f}, \ldots, e^{i 2(p-1) \pi f}\right)^{H}$, where $\otimes_{c}$ denotes the complex Kronecker product $\mathbf{A} \otimes_{c} \mathbf{B}$, i.e. the block matrix, the $(i, j)$ block element of which is $b_{i, j}^{*} \mathbf{A}^{6}$ and $\mathbf{K}$ is the vec-permutation matrix which transforms $\operatorname{Vec}(\mathbf{A})$ to $\operatorname{Vec}\left(\mathbf{A}^{T}\right)$ for any square matrix $\mathbf{A} . \quad S_{v}(f) \stackrel{\text { def }}{=} c_{u}\left|\sum_{q=0}^{Q} b_{q} e^{-i 2 \pi f q}\right|^{2}$ i.e., the spectral density of $v_{t}$ and $S^{\prime}{ }_{v}(f) \stackrel{\text { def }}{=}\left|c^{\prime}{ }_{u}\right|\left|\left(\sum_{q=0}^{Q} b_{q} e^{-i 2 \pi f q}\right)\left(\sum_{q=0}^{Q} b_{q} e^{+i 2 \pi f q}\right)\right|$. B denotes the $p \times(p+Q)$ filtering matrix $\left(\begin{array}{cccccc}b_{0} & b_{1} & \cdots & b_{Q} & & \\ & \ddots & & & \ddots & \\ & & & & & \\ & & b_{0} & b_{1} & \cdots & b_{Q}\end{array}\right)$.

Remark 1 In (2.6), $\operatorname{Cov}\left(\operatorname{Vec}\left(\mathbf{R}_{n}\right)\right)$ denotes $\mathrm{E}\left(\operatorname{Vec}\left(\mathbf{R}_{n}-\mathbf{R}\right) \operatorname{Vec}^{H}\left(\mathbf{R}_{n}-\mathbf{R}\right)\right)$. We note that

$$
\operatorname{Vec}^{T}\left(\mathbf{R}_{n}-\mathbf{R}\right)=\operatorname{Vec}^{H}\left(\mathbf{R}_{n}-\mathbf{R}\right) \mathbf{K}
$$

so $\mathrm{E}\left(\operatorname{Vec}\left(\mathbf{R}_{n}-\mathbf{R}\right) \operatorname{Vec}^{T}\left(\mathbf{R}_{n}-\mathbf{R}\right)\right)=\mathrm{E}\left(\operatorname{Vec}\left(\mathbf{R}_{n}-\mathbf{R}\right) \operatorname{Vec}^{H}\left(\mathbf{R}_{n}-\mathbf{R}\right)\right) \mathbf{K}$. Therefore the noncircular complex Gaussian asymptotic distribution of $\mathbf{R}_{n}$ is characterized by $\mathbf{C}_{R}$ only.

\footnotetext{
${ }^{5} \operatorname{Vec}($.$) is the "vectorization" operator that turns a matrix into a vector consisting of the columns of the matrix stacked$ one below another.

${ }^{6}$ This slightly unusual convention makes it easier to deal with complex matrices.
} 
Remark 2 We note that expression (2.7) of $\mathbf{C}_{R}$ obtained in the real case, cannot be deduced from expression (2.8) in the complex case as suggested by the Euler relation applied to relation (1.1):

$$
a_{k} \cos \left(2 \pi f_{k} t+\phi_{k}\right)=\frac{a_{k}}{2} e^{i \phi_{k}} e^{i 2 \pi f_{k} t}+\frac{a_{k}}{2} e^{-i \phi_{k}} e^{-i 2 \pi f_{k} t}
$$

In fact, if $S^{\prime}{ }_{v}(f), \mathbf{B}^{H}$ and $a_{k}^{2}\left[\mathbf{e}\left(f_{k}\right) \mathbf{e}^{H}\left(f_{k}\right) \otimes_{c} \mathbf{e}\left(f_{k}\right) \mathbf{e}^{H}\left(f_{k}\right)\right]$ are respectively replaced with $S_{v}(f), \mathbf{B}^{T}$ and $\left(\frac{a_{k}}{2}\right)^{2}\left[\mathbf{e}\left(f_{k}\right) \mathbf{e}^{H}\left(f_{k}\right) \otimes_{c} \mathbf{e}\left(f_{k}\right) \mathbf{e}^{H}\left(f_{k}\right)\right]+\left(\frac{a_{k}}{2}\right)^{2}\left[\mathbf{e}\left(-f_{k}\right) \mathbf{e}^{H}\left(-f_{k}\right) \otimes_{c} \mathbf{e}\left(-f_{k}\right) \mathbf{e}^{H}\left(-f_{k}\right)\right]$, an extra cross-term appears in (2.7). This surprising property is apparently contradictory, given the result that the asymptotic distribution of the sample covariance matrix does not depend on the phase model. In fact, the explanation comes from certain expressions proved in the complex case in the appendix (see footnote 8) being valid only if no frequencies are opposite. The fact that $\phi_{k}$ and $-\phi_{k}$, associated with $f_{k}$ and $-f_{k}$ are not independent, is irrelevant.

Remark 3 We note that for "very narrow-band" (i.e. the bandwidth is very small with respect to the sampling frequency) MA processes $v_{t}$, the expressions (2.7) and (2.8) are unbounded. For example, when $v_{t}$ tends to be white in $\left[f_{0}-b, f_{0}+b\right]$ with finite fixed power $c_{v}$, the expression (2.7) and (2.8) are not bounded with $b$, because $S_{v}(f)$ tends to $\left\{\begin{array}{lll}\frac{c_{v}}{2 b} & \text { for }\left|f-f_{0}\right| \leq b \\ 0 & \text { elsewhere }\end{array}\right.$ which implies that $\mathbf{C}_{R}$ contains the terms $\int_{-1 / 2}^{+1 / 2} S_{v}^{2}(f) d f$ that tend to $\frac{c_{v}^{2}}{2 b}$.

Since the matrix $\mathbf{R}$ is Toeplitz, the "accuracy" of its sample covariance estimate $\mathbf{R}_{n}$ which is non-Toeplitz should be improved by replacing it by its "Toeplitzed" estimate. This "Toeplitzation" also known as redundancy averaging in statistical signal and array processing applications [9], is carried out by averaging along the diagonals. The resulting estimate $\mathbf{R}_{n}^{\text {to }}$ is referred as the "Toeplitzed" estimated covariance matrix. Because this "Toeplitzation" operates a linear transform on $\mathbf{R}_{n}$, theorem 2 extends as follows:

Corollary $1 \operatorname{Vec}\left(\mathbf{R}_{n}\right)$ and $\operatorname{Vec}\left(\mathbf{R}_{n}^{\text {to }}\right)$ have the same asymptotic Gaussian distribution. It is characterized by the asymptotic distribution of the first column $\mathbf{r}_{n}$ of $\mathbf{R}_{n}$. In the real case [resp., in the complex case], we have:

$$
\sqrt{n}\left(\mathbf{r}_{n}-\mathbf{r}\right) \stackrel{\mathcal{L}}{\rightarrow} \mathcal{N}\left(\mathbf{0}, \mathbf{C}_{r}\right) \quad\left[\text { resp., } \mathcal{N}\left(\mathbf{0} ; \mathbf{C}_{r}, \mathbf{C}_{r}^{\prime}\right)\right] .
$$

Furthermore

$$
\begin{aligned}
\lim _{n \rightarrow \infty} \mathrm{E}\left(\mathbf{r}_{n}\right)=\mathbf{r} \text { and } \quad & \lim _{n \rightarrow \infty} n \mathrm{E}\left[\left(\mathbf{r}_{n}-\mathbf{r}\right)\left(\mathbf{r}_{n}-\mathbf{r}\right)^{T}\right]=\mathbf{C}_{r} \\
& {\left[\text { resp., } \quad \lim _{n \rightarrow \infty} n \mathrm{E}\left[\left(\mathbf{r}_{n}-\mathbf{r}\right)\left(\mathbf{r}_{n}-\mathbf{r}\right)^{H}\right]=\mathbf{C}_{r} \quad \lim _{n \rightarrow \infty} n \mathrm{E}\left[\left(\mathbf{r}_{n}-\mathbf{r}\right)\left(\mathbf{r}_{n}-\mathbf{r}\right)^{T}\right]=\mathbf{C}_{r}^{\prime}\right] }
\end{aligned}
$$

where $\mathbf{C}_{r}$, [resp., $\mathbf{C}_{r}, \mathbf{C}_{r}^{\prime}$ ] is defined in the real [resp., complex] case as:

$$
\mathbf{C}_{r}=\int_{-1 / 2}^{+1 / 2} S_{v}^{2}(f)\left[\mathbf{e}(f) \mathbf{e}^{H}(f)+\mathbf{e}(f) \mathbf{e}^{T}(f)\right] d f+\kappa_{u} \mathbf{B b b}^{T} \mathbf{B}^{T}
$$




$$
\begin{gathered}
+\frac{1}{2} \sum_{k=1}^{K} a_{k}^{2} S_{v}\left(f_{k}\right)\left[\mathbf{e}\left(f_{k}\right) \mathbf{e}^{H}\left(f_{k}\right)+\mathbf{e}\left(-f_{k}\right) \mathbf{e}^{H}\left(-f_{k}\right)+\mathbf{e}\left(f_{k}\right) \mathbf{e}^{H}\left(-f_{k}\right)+\mathbf{e}\left(-f_{k}\right) \mathbf{e}^{H}\left(f_{k}\right)\right] \\
\mathbf{C}_{r}=\int_{-1 / 2}^{+1 / 2}\left[S_{v}^{2}(f) \mathbf{e}(f) \mathbf{e}^{H}(f)+{S^{\prime}}_{v}^{2}(f) \mathbf{e}(f) \mathbf{e}^{T}(f)\right] d f+\kappa_{u} \mathbf{B} \mathbf{b} \mathbf{b}^{H} \mathbf{B}^{H}+2 \sum_{k=1}^{K} a_{k}^{2} S_{v}\left(f_{k}\right) \mathbf{e}\left(f_{k}\right) \mathbf{e}^{H}\left(f_{k}\right), \\
\mathbf{C}_{r}^{\prime}=\int_{-1 / 2}^{+1 / 2}\left[S_{v}^{2}(f) \mathbf{e}(f) \mathbf{e}^{T}(f)+{S^{\prime}}_{v}^{2}(f) \mathbf{e}(f) \mathbf{e}^{H}(f)\right] d f+\kappa_{u} \mathbf{B} \mathbf{b}^{*} \mathbf{b}^{H} \mathbf{B}^{T}+2 \sum_{k=1}^{K} a_{k}^{2} S_{v}\left(f_{k}\right) \mathbf{e}\left(f_{k}\right) \mathbf{e}^{T}\left(f_{k}\right),
\end{gathered}
$$

with $\mathbf{b}$ is the $(p+Q) \times 1$ vector $\left(b_{0}, \ldots, b_{Q}, 0, \ldots, 0\right)^{T}$.

Remark 3 In the complex case, we note that contrary to $\mathbf{R}_{n}$ (see remark 1), the asymptotic distribution of $\mathbf{r}_{n}$ is not caracterized by $\mathbf{C}_{r}$ only.

$$
\begin{cases}\text { Relation } \quad(2.11) & \text { reads componentwise with } \quad \mathbf{r}_{n} \stackrel{\text { def }}{=}\left(r_{n}^{0}, \ldots, r_{n}^{p-1}\right)^{T} \text { and } r_{b}^{i} \stackrel{\text { def }}{=} \\ b_{0} b_{i}+\ldots+b_{Q-i} b_{Q} & 0 \leq i \leq Q \\ 0 & Q<i \leq p-1 .\end{cases}
$$

$\lim _{n \rightarrow \infty} n \operatorname{Cov}\left(r_{n}^{i}, r_{n}^{j}\right)=2 \int_{-1 / 2}^{+1 / 2} S_{v}^{2}(f) \cos (2 \pi i f) \cos (2 \pi j f) d f+2 \sum_{k=1}^{K} a_{k}^{2} S_{v}^{2}\left(f_{k}\right) \cos \left(2 \pi i f_{k}\right) \cos \left(2 \pi j f_{k}\right)+\kappa_{u} r_{b}^{i} r_{b}^{j}, \quad i, j \geq 0$.

This extends the property given by [10] and by [3, Theorem 9.4] where $v_{t}$ is respectively a sequence of i.i.d. zero mean random variables with $\mathrm{E}\left(v_{t}^{4}\right)<\infty$ or a sequence of i.i.d. Gaussian distributed zero mean random variables.

\section{Application to estimation of sinusoidal frequencies}

Theorem 2 allows us to derive the asymptotic performance of most covariance-based sinusoidal frequencies estimation algorithms. With this aim, we adopt a functional analysis approach which consists in recognizing that the whole process of constructing an estimate $\mathbf{f}_{n}$ of $\mathbf{f} \stackrel{\text { def }}{=}\left(f_{1}, \ldots, f_{K}\right)^{T}$ is equivalent to defining a functional relationship linking this estimate $\mathbf{f}_{n}$ to the statistics $\mathbf{R}_{n}$ from which it is inferred. This functional dependence is denoted $\mathbf{f}_{n}=\operatorname{alg}\left(\mathbf{R}_{n}\right)$. Clearly, $\mathbf{f}=\operatorname{alg}(\mathbf{R})$ with $\mathbf{R}=\mathbf{E}(\mathbf{f}) \Delta \mathbf{E}^{H}(\mathbf{f})+c_{u} \mathbf{B B}^{H}{ }^{7}$, where $\mathbf{E}(\mathbf{f}) \stackrel{\text { def }}{=}\left(\mathbf{e}\left(f_{1}\right), \ldots, \mathbf{e}\left(f_{K}\right)\right)$ and $\boldsymbol{\Delta} \stackrel{\text { def }}{=} \operatorname{Diag}\left(a_{1}^{2}, \ldots, a_{K}^{2}\right)$. So the different algorithms $\operatorname{alg}($.$) constitute distinct extensions of the mapping \mathbf{R} \stackrel{\text { alg }}{\mapsto} \mathbf{f}$ generated by any unstructured Hermitian matrix $\mathbf{R}_{n}$. In the following, we consider "regular" algorithms. More specifically, we assume the following conditions:

1. The function $\operatorname{alg}($.$) is differentiable in a neighborhood of \mathbf{R}$, i.e., if $\mathbf{D}_{f, R}^{\mathrm{alg}} 8$ denotes the $K \times p^{2}$ matrix of this

\footnotetext{
${ }^{7}$ We consider complex case only, extension to the real case is straightforward.

${ }^{8}$ Expressions of $\mathbf{D}_{f, R}^{\text {alg }}$ are ordinarily deduced from perturbation calculus (see e.g. in [11] for the standard MUSIC algorithm).
} 
differential evaluated at point $\mathbf{R}$

$$
\operatorname{alg}(\mathbf{R}+\delta \mathbf{R})=\mathbf{f}+\mathbf{D}_{f, R}^{\mathrm{alg}} \operatorname{Vec}(\delta \mathbf{R})+o(\delta \mathbf{R})
$$

2. For any $\mathbf{f}$, any positive definite diagonal matrix $\boldsymbol{\Delta}$ and any $c_{u}$

$$
\operatorname{alg}\left(\mathbf{E}(\mathbf{f}) \boldsymbol{\Delta} \mathbf{E}^{H}(\mathbf{f})+c_{u} \mathbf{B B}^{H}\right)=\mathbf{f} .
$$

These two requirements are met for example by the high resolution second-order frequency estimators such as MUSIC, weighted MUSIC, Min-Norm, TAM and ESPRIT, which all assume that $v_{t}$ is white. With the conditions (3.1) and (3.2), the following result is proved in the Appendix.

Theorem $3 \sqrt{n}\left(\mathbf{f}_{n}-\mathbf{f}\right)$ converges in distribution to the zero-mean Gaussian distribution of covariance $\mathbf{C}_{f}$ which is invariant with respect to the distribution of the noise innovation.

$$
\sqrt{n}\left(\mathbf{f}_{n}-\mathbf{f}\right) \stackrel{\mathcal{L}}{\rightarrow} \mathcal{N}\left(\mathbf{0}, \mathbf{C}_{f}\right)
$$

with

$$
\mathbf{C}_{f}=\int_{-1 / 2}^{+1 / 2} \mathbf{D}_{f, R}^{\mathrm{alg}}\left[S_{v}^{2}(f)\left(\mathbf{e}(f) \mathbf{e}^{H}(f) \otimes_{c} \mathbf{e}(f) \mathbf{e}^{H}(f)\right)+{S^{\prime}}_{v}^{2}(f)\left(\mathbf{e}(f) \mathbf{e}^{T}(f) \otimes_{c} \mathbf{e}(f) \mathbf{e}^{T}(f)\right)\right]\left(\mathbf{D}_{f, R}^{\mathrm{alg}}\right)^{H} d f .
$$

So, although the asymptotic covariance of $\operatorname{Vec}\left(\mathbf{R}_{n}\right)$ is very sensitive to the distribution of $u_{t},\left(n \operatorname{Cov}\left(\operatorname{Vec}\left(\mathbf{R}_{n}\right)\right)\right.$ is unbounded for the super-Gaussian case under the constraint of fixed power because of $\kappa_{u}$ in expressions (2.7) and (2.8)), the asymptotic performance of most covariance-based sinusoidal frequencies estimators is invariant with respect to the distribution of the noise innovation. However, this asymptotic covariance $\mathbf{C}_{f}$ keeps unbounded for narrow-band noise of fixed power because of the term $\int_{-1 / 2}^{+1 / 2} S_{v}^{2}(f) d f$ in the expression (3.4). We note that theorem 3 extends a result given in [13] where explicit expressions for the covariance of the estimation errors associated with MUSIC and ESPRIT methods are derived for complex circular Gaussian white noise.

In the special case where the spectral density $S_{v}(f)$ is known up to a multiplicative constant, the whitening of the noise used classically in direction of arrival estimation (DOA) (see e.g. [14]) can be used to advantage. In this approach, after $v_{t}$ is whitened by a linear transformation applied to $\mathbf{x}_{t}$, any covariance-based DOA methods based on a calibrated array of generic steering vector in white noise (such as MUSIC, weighted MUSIC, Min-Norm,...) can be used. In these circumstances, it makes sense to study the influence of the spectrum of $v_{t}$ and the selected linear transformation on the performance of this sinusoidal frequency estimator. Considering our functional analysis, theorem 3 answers this question. The process $v_{t}$ is whitened using the Cholesky decomposition $\mathbf{L}^{H} \mathbf{L}$ of $\left(\mathbf{B B}^{H}\right)^{-1}$ 
(see e.g. [15, relation 1.7.19]) and any unitary matrix $\mathbf{Q}$ :

$$
\left(\mathbf{B B}^{H}\right)^{-1}=\mathbf{L}^{\prime H} \mathbf{L}^{\prime} \quad \text { with } \quad \mathbf{L}^{\prime} \stackrel{\text { def }}{=} \mathbf{Q} \mathbf{L}
$$

and the covariance matrix of $\mathbf{x}_{t}$ becomes:

$$
\mathbf{R}^{\prime}=\mathbf{L}^{\prime} \mathbf{E}(\mathbf{f}) \boldsymbol{\Delta}\left(\mathbf{L}^{\prime} \mathbf{E}(\mathbf{f})\right)^{H}+c_{u} \mathbf{I}
$$

If alg (.) denotes an algorithm based on generic steering vector and white noise assumption, the sinusoidal frequencies are estimated with the following scheme:

$$
\mathbf{R}_{n} \mapsto \mathbf{R}_{n}^{\prime} \stackrel{\text { def }}{=} \mathbf{L}^{\prime} \mathbf{R}_{n} \mathbf{L}^{\prime H} \stackrel{\text { alg }}{\mapsto} \mathbf{f}_{n} \Rightarrow \mathbf{R}_{n} \stackrel{\text { alg' }}{\mapsto} \mathbf{f}_{n}
$$

Applying the chain differential rule, theorem 3 applies in this situation by replacing in (3.4), $\mathbf{D}_{f, R}^{\text {alg }}$ by $\mathbf{D}_{f, R}^{\text {alg' }}=$ $\mathbf{D}_{f, R^{\prime}}^{\text {alg }}\left(\mathbf{L}^{\prime} \otimes_{c} \mathbf{L}^{\prime}\right)$, because $\operatorname{Vec}\left(\mathbf{R}_{n}^{\prime}\right)=\left(\mathbf{L}^{\prime} \otimes_{c} \mathbf{L}^{\prime}\right) \operatorname{Vec}\left(\mathbf{R}_{n}\right)$.

\section{A Proof of theorems}

The complex case is considered only as the same approach may be used for the real case.

\section{A.1 Proof of theorem (1)}

First of all, $\sqrt{n}\left(m_{n}-m\right)$ is decomposed as

$$
\sqrt{n}\left(m_{n}-m\right)=\sum_{k=1}^{K} a_{k} e^{i \phi_{k}} \frac{1}{\sqrt{n}}\left(\sum_{t=1}^{n} e^{i 2 \pi f_{k} t}\right)+\frac{1}{\sqrt{n}} \sum_{t=1}^{n} v_{t} .
$$

Because $\left|\sum_{t=1}^{n} e^{i 2 \pi f_{k} t}\right|=\left|\frac{\sin \pi n f_{k}}{\sin \pi f_{k}}\right|$ is bounded, the first term of (A.1) converges almost surely to 0 when $n \rightarrow \infty$ whatever the phase model. Thus, we can consider the term $\frac{1}{\sqrt{n}} \sum_{t=1}^{n} v_{t}$ only, in the study of the convergence in distribution of $\sqrt{n}\left(m_{n}-m\right)$. Let $y_{t} \stackrel{\text { def }}{=} w^{*} v_{t}+w v_{t}^{*} \cdot y_{t}$ is a real stationary $Q$-dependent sequence of random variables with mean zero and correlation $\gamma_{k}$ with

$$
\gamma_{k}=\sum_{q=0}^{Q} c_{u}|w|^{2}\left(b_{k+q}^{*} b_{q}+b_{k+q} b_{q}^{*}\right)+c_{u}^{\prime} w^{* 2} b_{k+q} b_{q}+{c^{\prime}}_{u}^{*} w^{2} b_{k+q}^{*} b_{q}^{*} .
$$

The conditions of $\left[2\right.$, theorem 6.4.2] are fulfilled. Thus, $\frac{1}{\sqrt{n}} \sum_{t=1}^{n} y_{t}$ converges in distribution to the zeromean real Gaussian distribution of variance $c_{y}=\gamma_{0}+2 \sum_{q=1}^{Q} \gamma_{q}$ and $\lim _{n \rightarrow \infty} n \operatorname{Var}\left(\frac{1}{n} \sum_{t=1}^{n} y_{t}\right)=c_{y}$ with $c_{y}=2|w|^{2} c_{u}\left|\sum_{q=0}^{Q} b_{q}\right|^{2}+w^{* 2} c_{u}^{\prime}\left(\sum_{q=0}^{Q} b_{q}\right)^{2}+w^{2} c_{u}^{\prime *}\left(\sum_{q=0}^{Q} b_{q}^{*}\right)^{2}$. The theorem follows thanks to (2.1). 


\section{A.2 Proof of theorem (2)}

Because $\frac{1}{n} \sum_{t=1}^{n} v_{t}$ converges in probability to 0 (see e.g. [2, proposition 6.3 .10$]$ ), $\mathbf{m}_{n}$ converges in probability to m. Using a classical result (e.g. deduced from [2, proposition 6.3.4] and [2, proposition 6.3.7]), we can deduce that studying the asymptotic distribution of $\mathbf{R}_{n}$, boils downs to studying the asymptotic distribution of $\mathbf{R}_{n}^{\prime} \stackrel{\text { def }}{=}$ $\frac{1}{n} \sum_{t=1}^{n}\left(\mathbf{x}_{t}-\mathbf{m}\right)\left(\mathbf{x}_{t}-\mathbf{m}\right)^{H}$. Then, using $\operatorname{Vec}\left(\mathbf{a b}^{H}\right)=\mathbf{a} \otimes_{c} \mathbf{b}, \operatorname{Vec}\left(\mathbf{R}_{n}^{\prime}-\mathbf{R}\right)$ is decomposed as

$$
\operatorname{Vec}\left(\mathbf{R}_{n}^{\prime}-\mathbf{R}\right)=\frac{1}{n} \sum_{t=1}^{n}\left(\mathbf{z}_{t}^{1}+\mathbf{z}_{t}^{2}\right)
$$

with $\mathbf{z}_{t}^{1} \stackrel{\text { def }}{=} \mathbf{c}_{t} \otimes_{c} \mathbf{v}_{t}+\mathbf{v}_{t} \otimes_{c} \mathbf{c}_{t}+\mathbf{v}_{t} \otimes_{c} \mathbf{v}_{t}-\operatorname{Vec}\left(\mathbf{R}_{v}\right)$ and $\mathbf{z}_{t}^{2} \stackrel{\text { def }}{=} \sum_{1 \leq k \neq k^{\prime} \leq K} a_{k} a_{k^{\prime}} e^{i\left(\phi_{k}-\phi_{k^{\prime}}\right)} e^{i 2 \pi\left(f_{k}-f_{k^{\prime}}\right) t} \mathbf{e}\left(f_{k}\right) \otimes_{c} \mathbf{e}\left(f_{k^{\prime}}\right)$ where $\mathbf{c}_{t} \stackrel{\text { def }}{=} \sum_{k=1}^{K} a_{k} e^{i \phi_{k}} e^{i 2 \pi f_{k} t} \mathbf{e}\left(f_{k}\right), \mathbf{v}_{t} \stackrel{\text { def }}{=}\left(v_{t}, v_{t-1}, \ldots, v_{t-p+1}\right)^{T}$ and $\mathbf{R}_{v} \stackrel{\text { def }}{=} \mathrm{E}\left(\mathbf{v}_{t} \mathbf{v}_{t}^{H}\right)$. Because

$$
\left\|\left|\sqrt{n} \frac{1}{n} \sum_{t=1}^{n} \mathbf{z}_{t}^{2}\left\|\leq \frac{1}{\sqrt{n}} \sum_{1 \leq k \neq k^{\prime} \leq K} a_{k} a_{k^{\prime}}\left|\frac{\sin \left(\pi n\left(f_{k}-f_{k^{\prime}}\right)\right.}{\sin \left(\pi\left(f_{k}-f_{k^{\prime}}\right)\right.}\right|\right\| \mathbf{e}\left(f_{k}\right) \otimes_{c} \mathbf{e}\left(f_{k^{\prime}}\right) \|,\right.\right.
$$

$\sqrt{n} \frac{1}{n} \sum_{t=1}^{n} \mathbf{z}_{t}^{2}$ converges almost surely to $\mathbf{0}$ when $n \rightarrow \infty$. Thus, we can consider the term $\frac{\sqrt{n}}{n} \sum_{t=1}^{n} \mathbf{z}_{t}^{1}$ alone, in the study of the convergence in distribution of $\sqrt{n} \operatorname{Vec}\left(\mathbf{R}_{n}^{\prime}-\mathbf{R}\right)$. To prove the convergence in distribution of $\sqrt{n} \frac{1}{n} \sum_{t=1}^{n} \mathbf{z}_{t}^{1}$ to a zero-mean noncircular complex Gaussian distribution, we consider the associated scalar real random variable $y_{t} \stackrel{\text { def }}{=} \mathbf{w}^{H} \mathbf{z}_{t}^{1}+\left(\mathbf{w}^{H} \mathbf{z}_{t}^{1}\right)^{H}$ (see definition of Section 2). The conditional distribution of $\left(y_{t}\right)_{t=1, \ldots, n}$ given the phases $\left(\phi_{k}\right)_{k=1, \ldots, K}$ is a zero-mean real distribution of $L$-dependent (with $L=p+Q$ ) but not strictly stationary random variables because $y_{t}$ are not identically distributed. As such, the conditions of [2, theorem 6.4.2] are no longer fully satisfied.

To prove theorem 2, we continue to make use of [2, theorem 6.4.2] by some modifications of its proof. Following this proof, we must consider first, the limit of $n \operatorname{Var}\left(\frac{1}{n} \sum_{t=1}^{n} y_{t} / \phi\right)$ when $n \rightarrow \infty$. Thanks to (2.9), this expression may be written as:

$$
2 \mathbf{w}^{H}\left[\frac{1}{n} \sum_{1 \leq s, t \leq n} \mathrm{E}\left(\mathbf{z}_{s}^{1} \mathbf{z}_{t}^{1^{H}} / \phi\right)\right] \mathbf{w}+\mathbf{w}^{H}\left[\frac{1}{n} \sum_{1 \leq s, t \leq n} \mathrm{E}\left(\mathbf{z}_{s}^{1} \mathbf{z}_{t}^{1^{H}} / \phi\right)\right] \mathbf{K} \mathbf{w}^{*}+\mathbf{w}^{T}\left[\frac{1}{n} \sum_{1 \leq s, t \leq n} \mathrm{E}\left(\mathbf{z}_{s}^{1} \mathbf{z}_{t}^{1}{ }^{H} / \phi\right)\right]^{*} \mathbf{K} \mathbf{w}(\mathrm{A}
$$

where $\frac{1}{n} \sum_{1 \leq s, t \leq n} \mathrm{E}\left(\mathbf{z}_{s}^{1} \mathbf{z}_{t}^{1 H} / \phi\right)=\mathbf{T}_{a}+\mathbf{T}_{b}+\mathbf{T}_{c}$ with

$$
\begin{aligned}
& \mathbf{T}_{a} \stackrel{\text { def }}{=} \frac{1}{n} \sum_{1 \leq s, t \leq n} \mathrm{E}\left(\left(\mathbf{v}_{s} \otimes_{c} \mathbf{c}_{s}\right)\left(\mathbf{c}_{t}^{H} \otimes_{c} \mathbf{v}_{t}^{H}\right) / \phi\right)+\mathrm{E}\left(\left(\mathbf{c}_{s} \otimes_{c} \mathbf{v}_{s}\right)\left(\mathbf{v}_{t}^{H} \otimes_{c} \mathbf{c}_{t}^{H}\right) / \phi\right), \\
& \mathbf{T}_{b} \stackrel{\text { def }}{=} \frac{1}{n} \sum_{1 \leq s, t \leq n} \mathrm{E}\left(\left(\mathbf{v}_{s} \otimes_{c} \mathbf{c}_{s}\right)\left(\mathbf{v}_{t}^{H} \otimes_{c} \mathbf{c}_{t}^{H}\right) / \phi\right)+\mathrm{E}\left(\left(\mathbf{c}_{s} \otimes_{c} \mathbf{v}_{s}\right)\left(\mathbf{c}_{t}^{H} \otimes_{c} \mathbf{v}_{t}^{H}\right) / \phi\right), \\
& \mathbf{T}_{c} \stackrel{\text { def }}{=} \frac{1}{n} \sum_{1 \leq s, t \leq n} \mathrm{E}\left(\left(\mathbf{v}_{s} \otimes_{c} \mathbf{v}_{s}-\operatorname{Vec}\left(\mathbf{R}_{v}\right)\left(\mathbf{v}_{t} \otimes_{c} \mathbf{v}_{t}-\operatorname{Vec}\left(\mathbf{R}_{v}\right)\right)^{H} .\right.\right.
\end{aligned}
$$

Thanks to the following property of the vec-permutation matrix $\mathbf{K}$

$$
\left(\mathbf{a}^{H} \otimes_{c} \mathbf{b}^{H}\right) \mathbf{K}=\mathbf{b}^{T} \otimes_{c} \mathbf{a}^{T}
$$


$\mathrm{E}\left(\left(\mathbf{v}_{s} \otimes_{c} \mathbf{c}_{s}\right)\left(\mathbf{c}_{t}^{H} \otimes_{c} \mathbf{v}_{t}^{H}\right) / \phi\right)+\mathrm{E}\left(\left(\mathbf{c}_{s} \otimes_{c} \mathbf{v}_{s}\right)\left(\mathbf{v}_{t}^{H} \otimes_{c} \mathbf{c}_{t}^{H}\right) / \phi\right)=\left[\left(\mathrm{E}\left(\mathbf{v}_{s} \mathbf{v}_{t}^{T}\right) \otimes_{c} \mathbf{c}_{s} \mathbf{c}_{t}^{T}\right)+\left(\mathbf{c}_{s} \mathbf{c}_{t}^{T} \otimes_{c} \mathrm{E}\left(\mathbf{v}_{s} \mathbf{v}_{t}^{T}\right)\right)\right] \mathbf{K}$, thus the first term of $\mathbf{T}_{a}$ becomes:

$$
\sum_{l=-p-Q+1}^{p+Q-1} \mathbf{R}_{v}^{\prime}(l) \otimes_{c}\left(\sum_{1 \leq k, k^{\prime} \leq K} a_{k} a_{k^{\prime}} e^{i\left(\phi_{k}+\phi_{k^{\prime}}\right)} e^{-i 2 \pi f_{k^{\prime}} l}\left[\frac{1}{n} \sum_{s \in S_{n, l}} e^{i 2 \pi\left(f_{k}+f_{k^{\prime}}\right) s}\right] \mathbf{e}\left(f_{k}\right) \mathbf{e}^{T}\left(f_{k^{\prime}}\right)\right) \mathbf{K}
$$

with $\mathbf{R}_{v}^{\prime}(l) \stackrel{\text { def }}{=} \mathrm{E}\left(\mathbf{v}_{s} \mathbf{v}_{s-l}^{T}\right)$ and $S_{n, l}$ is the set $\{s, 1 \leq s \leq n-l$ for $l \geq 0$ or $-l+1 \leq s \leq n$ for $l \leq 0\}$. Because $\lim _{n \rightarrow \infty}\left|\frac{1}{n} \sum_{s \in S_{n, l}} e^{i 2 \pi\left(f_{k}+f_{k^{\prime}}\right) s}\right|=0^{9}$, the first term of $\mathbf{T}_{a}$ and therefore the term $\mathbf{T}_{a}$ tends to $\mathbf{O}$ when $n \rightarrow \infty$. As $\mathrm{E}\left(\left(\mathbf{v}_{s} \otimes_{c} \mathbf{c}_{s}\right)\left(\mathbf{v}_{t}^{H} \otimes_{c} \mathbf{c}_{t}^{H}\right) / \phi\right)+\mathrm{E}\left(\left(\mathbf{c}_{s} \otimes_{c} \mathbf{v}_{s}\right)\left(\mathbf{c}_{t}^{H} \otimes_{c} \mathbf{v}_{t}^{H}\right) / \phi\right)=\left(\mathrm{E}\left(\mathbf{v}_{s} \mathbf{v}_{t}^{H}\right) \otimes_{c} \mathbf{c}_{s} \mathbf{c}_{t}^{H}\right)+\left(\mathbf{c}_{s} \mathbf{c}_{t}^{H} \otimes_{c} \mathrm{E}\left(\mathbf{v}_{s} \mathbf{v}_{t}^{H}\right)\right)$, the first term of $\mathbf{T}_{b}$ becomes:

$$
\sum_{l=-p-Q+1}^{p+Q-1} \mathbf{R}_{v}(l) \otimes_{c}\left(\sum_{1 \leq k, k^{\prime} \leq K} a_{k} a_{k^{\prime}} e^{i\left(\phi_{k}-\phi_{k^{\prime}}\right)} e^{i 2 \pi f_{k^{\prime}} l}\left[\frac{1}{n} \sum_{s \in S_{n, l}} e^{i 2 \pi\left(f_{k}-f_{k^{\prime}}\right) s}\right] \mathbf{e}\left(f_{k}\right) \mathbf{e}^{H}\left(f_{k^{\prime}}\right)\right)
$$

with $\mathbf{R}_{v}(l) \stackrel{\text { def }}{=} \mathrm{E}\left(\mathbf{v}_{s} \mathbf{v}_{s-l}^{H}\right)$. Because $\lim _{n \rightarrow \infty}\left|\frac{1}{n} \sum_{s \in S_{n, l}} e^{i 2 \pi\left(f_{k}-f_{k^{\prime}}\right) s}\right|=0$ for $f_{k} \neq f_{k^{\prime}}$ and 1 for $f_{k}=f_{k^{\prime}}$, the first term of $\mathbf{T}_{b}$ tends to $\sum_{1 \leq k \leq K} a_{k}^{2}\left(\sum_{l=-p-Q+1}^{p+Q-1} \mathbf{R}_{v}(l)\right) e^{-i 2 \pi f_{k} l} \otimes_{c} \mathbf{e}\left(f_{k}\right) \mathbf{e}^{H}\left(f_{k}\right)$ when $n \rightarrow \infty$. With $\left(\sum_{l=-p-Q+1}^{p+Q-1} \mathbf{R}_{v}(l)\right) e^{-i 2 \pi f_{k} l}=S_{v}\left(f_{k}\right) \mathbf{e}\left(f_{k}\right) \mathbf{e}^{H}\left(f_{k}\right)$, the term $\mathbf{T}_{b}$ tends to

$$
2 \sum_{1 \leq k \leq K} a_{k}^{2} S_{v}\left(f_{k}\right)\left[\mathbf{e}\left(f_{k}\right) \mathbf{e}^{H}\left(f_{k}\right) \otimes_{c} \mathbf{e}\left(f_{k}\right) \mathbf{e}^{H}\left(f_{k}\right)\right] .
$$

Then, because $\mathbf{v}_{t}=\mathbf{B} \mathbf{u}_{t}$ with $\mathbf{u}_{t} \stackrel{\text { def }}{=}\left(u_{t}, u_{t-1, \ldots, u_{t-p-Q+1}}\right)^{T}$, the term $\mathbf{T}_{c}$ becomes:

$$
\left(\mathbf{B} \otimes_{c} \mathbf{B}\right) n \operatorname{Cov}\left(\operatorname{Vec}\left(\mathbf{R}_{n}^{u}\right)\right)\left(\mathbf{B}^{H} \otimes_{c} \mathbf{B}^{H}\right)
$$

with $\mathbf{R}_{n}^{u} \stackrel{\text { def }}{=} \frac{1}{n} \sum_{t=1}^{n} \mathbf{u}_{t} \mathbf{u}_{t}^{H} \cdot\left[n \operatorname{Cov}\left(\operatorname{Vec}\left(\mathbf{R}_{n}^{u}\right)\right)\right]_{(j-1)(p+Q)+i,(l-1)(p+Q)+k}$ then becomes:

$$
\frac{1}{n} \sum_{1 \leq s, t \leq n} \mathrm{E}\left(u_{s-i+1} u_{s-j+1}^{*} u_{t-k+1}^{*} u_{t-l+1}\right)-\mathrm{E}\left(u_{s-i+1} u_{s-j+1}^{*}\right) \mathrm{E}\left(u_{t-k+1}^{*} u_{t-l+1}\right)
$$

By definition of $\operatorname{Cum}\left(u_{s-i+1}, u_{s-j+1}^{*}, u_{t-k+1}^{*}, u_{t-l+1}\right),($ A.6) is decomposed as

$$
\mathrm{E}\left(u_{s-i+1} u_{t-k+1}^{*}\right) \mathrm{E}\left(u_{s-j+1} u_{t-k+1}^{*}\right)+\mathrm{E}\left(u_{s-i+1} u_{t-l+1}\right) \mathrm{E}\left(u_{s-j+1}^{*} u_{t-k+1}^{*}\right)+\mathrm{Cum}\left(u_{s-i+1}, u_{s-j+1}^{*}, u_{t-k+1}^{*}, u_{t-l+1}\right) .
$$

These three terms are respectively equal to

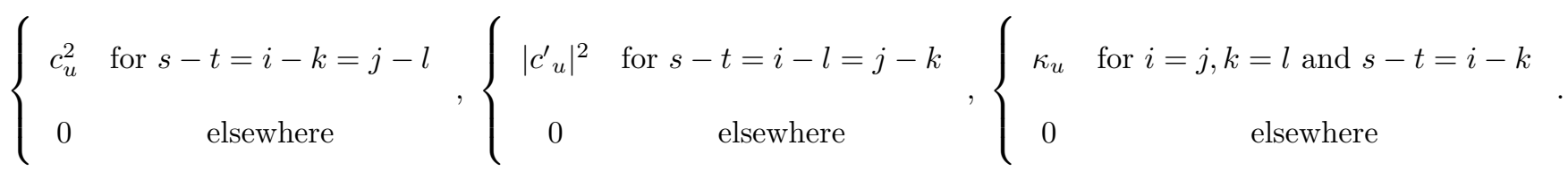

Consequently, $\lim _{n \rightarrow \infty}\left[n \operatorname{Cov}\left(\operatorname{Vec}\left(\mathbf{R}_{n}^{u}\right)\right)\right]_{(j-1)(p+Q)+i,(l-1)(p+Q)+k}$ is defined and decomposed as

$$
\left\{\begin{array}{cc}
c_{u}^{2} & \text { for } i-k=j-l \\
0 & \text { elsewhere }
\end{array}+\left\{\begin{array}{cc}
\left|c^{\prime}{ }_{u}\right|^{2} & \text { for } i-l=j-k \\
0 & \text { elsewhere }
\end{array}+\left\{\begin{array}{cc}
\kappa_{u} & \text { for } i=j, k=l \\
0 & \text { elsewhere }
\end{array}\right.\right.\right.
$$

\footnotetext{
${ }^{9}$ Except for the specific case where two frequencies $f_{k}$ are opposite.
} 
whose associated matrix is

$$
c_{u}^{2} \int_{-1 / 2}^{+1 / 2}\left[\mathbf{e}(f) \mathbf{e}^{H}(f) \otimes_{c} \mathbf{e}(f) \mathbf{e}^{H}(f)\right] d f+\left|c^{\prime}{ }_{u}\right|^{2} \int_{-1 / 2}^{+1 / 2}\left[\mathbf{e}(f) \mathbf{e}^{H}(f) \otimes_{c} \mathbf{e}(f) \mathbf{e}^{H}(f)\right] \mathbf{K} d f+\kappa_{u} \operatorname{Vec}(\mathbf{I}) \operatorname{Vec}^{T}(\mathbf{I}) .
$$

Then

$$
\begin{gathered}
\left(\mathbf{B} \otimes_{c} \mathbf{B}\right)\left[\int_{-1 / 2}^{+1 / 2}\left[\mathbf{e}(f) \mathbf{e}^{H}(f) \otimes_{c} \mathbf{e}(f) \mathbf{e}^{H}(f)\right] d f\right]\left(\mathbf{B}^{H} \otimes_{c} \mathbf{B}^{H}\right)=\int_{-1 / 2}^{+1 / 2}\left(\mathbf{B e}(f) \mathbf{e}^{H}(f) \mathbf{B}^{H}\right) \otimes_{c}\left(\mathbf{B e}(f) \mathbf{e}^{H}(f) \mathbf{B}^{H}\right) d f \\
\left(\mathbf{B} \otimes_{c} \mathbf{B}\right)\left[\int_{-1 / 2}^{+1 / 2}\left[\mathbf{e}(f) \mathbf{e}^{H}(f) \otimes_{c} \mathbf{e}(f) \mathbf{e}^{H}(f)\right] \mathbf{K} d f\right]\left(\mathbf{B}^{H} \otimes_{c} \mathbf{B}^{H}\right)=\int_{-1 / 2}^{+1 / 2}\left(\mathbf{B e}(f) \mathbf{e}^{H}(f) \mathbf{B}^{T}\right) \otimes_{c}\left(\mathbf{B e}(f) \mathbf{e}^{H}(f) \mathbf{B}^{T}\right) d f
\end{gathered}
$$

thanks to (A.3) with $c_{u} \mathbf{B e}(f) \mathbf{e}^{H}(f) \mathbf{B}^{H}=S_{v}(f) \mathbf{e}(f) \mathbf{e}^{H}(f)$ and $\left|c^{\prime}{ }_{u}\right| \mathbf{B e}(f) \mathbf{e}^{H}(f) \mathbf{B}^{T}=S^{\prime}{ }_{v}(f) \mathbf{e}(f) \mathbf{e}^{H}(f)$. And then

$$
\kappa_{u}\left(\mathbf{B} \otimes_{c} \mathbf{B}\right) \operatorname{Vec}(\mathbf{I}) \operatorname{Vec}^{T}(\mathbf{I})\left(\mathbf{B}^{H} \otimes_{c} \mathbf{B}^{H}\right)=\kappa_{u} \operatorname{Vec}\left(\mathbf{B B}^{H}\right) \operatorname{Vec}^{H}\left(\mathbf{B B}^{H}\right)
$$

thanks to $\operatorname{Vec}(\mathbf{A B C})=\left(\mathbf{A} \otimes_{c} \mathbf{C}^{H}\right) \operatorname{Vec}(\mathbf{B})$. Putting together the limits of term $\mathbf{T}_{b}$ and of term $\mathbf{T}_{c}$, $\lim _{n \rightarrow \infty} \frac{1}{n} \sum_{1 \leq s, t \leq n} \mathrm{E}\left(\mathbf{z}_{s}^{1} \mathbf{z}_{t}^{1}{ }^{H} / \phi\right)$ is defined and does not depend on the phases $\phi_{k}$. Thanks to (A.2) and (2.1), we get expression (2.8) irrespective of the phase model.

Then, following the proof of [2, theorem 6.4.2], the application of the classical central limit (CL) theorem (e.g. $[2$, theorem 6.4.1]) to the sum:

$$
\frac{1}{\sqrt{n}} \sum_{t=1}^{r} y_{t, k} \quad \text { with } \quad y_{t, k} \stackrel{\text { def }}{=} y_{(t-1) k+1}+\ldots+y_{t k-L}, \quad \text { where } \quad r \stackrel{\text { def }}{=}\lfloor n / k\rfloor \text { and } k \text { fixed with } k>L
$$

for $n \rightarrow \infty$ is not valid because $\left(y_{t, k}\right)_{t=1, \ldots, r}$ are zero-mean independent but not identically distributed random variables. We replace the classical CL theorem by the Lyapunov theorem (see e.g. [8, p. 371]) by verifying the following Lyapunov's condition [8, relation (27.16)] with $\delta=2$ :

$$
\lim _{r \rightarrow \infty} \frac{\sum_{t=1}^{r} \mathrm{E}\left(y_{t, k}^{2+2} / \phi\right)}{\left(\sqrt{\sum_{t=1}^{r} \mathrm{E}\left(y_{t, k}^{2} / \phi\right)}\right)^{2+2}}=0
$$

As $\frac{1}{n} \sum_{t=1}^{r} \mathrm{E}\left(y_{t, k}^{2} / \phi\right)=n \operatorname{Var}\left(\frac{1}{n} \sum_{t=1}^{r} y_{t, k} / \phi\right)$, we get after similar manipulations as previously that $\sum_{t=1}^{r} \mathrm{E}\left(y_{t, k}^{2} / \phi\right) \propto n$ when $n \rightarrow \infty$. On the other hand, $\frac{1}{n} \sum_{t=1}^{r} \mathrm{E}\left(y_{t, k}^{4} / \phi\right)$ can be decomposed as the sum of terms

$$
c_{a, b, c, d}(\mathbf{w}) \frac{1}{n} \sum_{t=1}^{r}\left(\sum_{1 \leq l, l^{\prime}, l^{\prime \prime}, l^{\prime \prime \prime} \leq k-L} \mathrm{E}\left(\left(\mathbf{z}_{(t-1) k+l}^{1}\right)_{a}^{s_{a}}\left(\mathbf{z}_{(t-1) k+l^{\prime}}^{1}\right)_{b}^{s_{b}}\left(\mathbf{z}_{(t-1) k+l^{\prime \prime}}^{1}\right)_{c}^{s_{c}}\left(\mathbf{z}_{(t-1) k+l^{\prime \prime \prime}}^{1}\right)_{d}^{s_{d}} / \phi\right)\right),
$$

for $0 \leq a, b, c, d \leq p^{2}$, where $c_{a, b, c, d}(\mathbf{w})$ is an appropriate function of $\mathbf{w}$ and where $\left(\mathbf{y}_{(t-1) k+l}^{1}\right)_{i}^{s_{i}}$ denotes the $i$-th component of $\mathbf{y}_{(t-1) k+l}^{1}$ which is conjugated for certain indices. Because an examination of the term (A.8) shows that it has a limit when $n \rightarrow \infty, \sum_{t=1}^{r} \mathrm{E}\left(y_{t, k}^{4} / \phi\right) \propto n$ when $n \rightarrow \infty$. So Lyapounov's condition (A.7) with $\delta=2$ 
is proved. Therefore the conditional real scalar random variable $\frac{\sum_{t=1}^{r} y_{t, k}}{\sqrt{\sum_{t=1}^{r} \mathrm{E}\left(y_{t, k}^{2} / \phi\right)}}$ converges in distribution to the zero-mean, unit variance real Gaussian distribution when $r \rightarrow \infty$.

Finally, incorporating the other elements of the proof of [2, theorem 6.4.2], $\sqrt{n} \frac{1}{n} \sum_{t=1}^{n} y_{t}$ converges in distribution to the zero-mean, Gaussian distribution of variance $2 \mathbf{w}^{H} \mathbf{C}_{R} \mathbf{w}+\mathbf{w}^{H} \mathbf{C}_{R} \mathbf{K w}^{*}+\mathbf{w}^{T} \mathbf{C}_{R}^{*} \mathbf{K w}$ with $\mathbf{C}_{R}$ given by (2.8). And by application of the Cramer-Wold theorem [8, theorem 29.4], the complex random vector $\sqrt{n} \frac{1}{n} \sum_{t=1}^{n} \mathbf{z}_{t}^{1}$ converges in distribution to the zero-mean complex noncircular Gaussian distribution $\mathcal{N}\left(\mathbf{0} ; \mathbf{C}_{R}, \mathbf{C}_{R} \mathbf{K}\right)$.

\section{A.3 Proof of corollary (1)}

Thanks to the "Toeplitzation" projection matrix $\mathbf{T}_{o}, \operatorname{Vec}\left(\mathbf{R}_{n}^{\text {to }}\right)=\mathbf{T}_{o} \operatorname{Vec}\left(\mathbf{R}_{n}\right)$. Therefore, theorem 2 extends to $\mathbf{R}_{n}^{\text {to }}$ with the asymptotic covariance matrix $\mathbf{C}_{R}^{\text {to }}=\mathbf{T}_{o} \mathbf{C}_{R} \mathbf{T}_{o}$. Because

$$
\mathbf{e}\left(f_{k}\right) \mathbf{e}^{+}\left(f_{l}\right) \otimes_{c} \mathbf{e}\left(f_{k}\right) \mathbf{e}^{+}\left(f_{l}\right)=\left(\mathbf{e}\left(f_{k}\right) \otimes_{c} \mathbf{e}\left(f_{k}\right)\right)\left(\mathbf{e}^{+}\left(f_{l}\right) \otimes_{c} \mathbf{e}^{+}\left(f_{l}\right)\right) \quad \text { with }+\stackrel{\text { def }}{=} T \text { or } H
$$

and $\mathbf{e}\left(f_{i}\right) \otimes_{c} \mathbf{e}\left(f_{i}\right)=\operatorname{Vec}\left(\mathbf{e}\left(f_{i}\right) \mathbf{e}^{H}\left(f_{i}\right)\right)$ with $\mathbf{e}\left(f_{i}\right) \mathbf{e}^{H}\left(f_{i}\right)$ is a Toeplitz matrix,

$$
\mathbf{T}_{o}\left(\mathbf{e}\left(f_{k}\right) \mathbf{e}^{+}\left(f_{l}\right) \otimes_{c} \mathbf{e}\left(f_{k}\right) \mathbf{e}^{+}\left(f_{l}\right)\right) \mathbf{T}_{o}=\mathbf{e}\left(f_{k}\right) \mathbf{e}^{+}\left(f_{l}\right) \otimes_{c} \mathbf{e}\left(f_{k}\right) \mathbf{e}^{+}\left(f_{l}\right)
$$

Then, because $\mathbf{B B}^{+}$is also a Toeplitz matrix, relation $\mathbf{T}_{o} \mathbf{C}_{R} \mathbf{T}_{o}=\mathbf{C}_{R}$ is proved. Therefore the "Toeplitzation" does not improve the covariance estimate and the expressions of $\mathbf{C}_{r}$ and $\mathbf{C}_{r}^{\prime}$ are given by the blocks (1,1) of $\mathbf{C}_{R}$ and $\mathbf{C}_{R}^{\prime}$ respectively.

\section{A.4 Proof of theorem (3)}

From the regularity condition (3.1), the asymptotic behaviors of $\mathbf{f}_{n}$ and $\mathbf{R}_{n}$ are directly related. The standard result on regular functions of asymptotically normal statistics (see e.g. [12, theorem, p. 122]) applies. So (3.3) holds with $\mathbf{C}_{f}=\mathbf{D}_{f, R}^{\text {alg }} \mathbf{C}_{R}\left(\mathbf{D}_{f, R}^{\text {alg }}\right)^{H}$. Furthermore, this closed-form expression simplifies if the conditions (1) and (2) are taken into account.

$$
\begin{aligned}
\mathbf{f} & =\operatorname{alg}\left(\mathbf{E}(\mathbf{f})(\boldsymbol{\Delta}+\delta \boldsymbol{\Delta}) \mathbf{E}^{H}(\mathbf{f})+\left(c_{u}+\delta c_{u}\right) \mathbf{B} \mathbf{B}^{H}\right) \\
& =\mathbf{f}+\mathbf{D}_{f, R}^{\mathrm{alg}} \operatorname{Vec}\left(\mathbf{E}(\mathbf{f}) \delta \boldsymbol{\Delta} \mathbf{E}^{H}(\mathbf{f})+\delta c_{u} \mathbf{B B}^{H}\right)+o(\delta \boldsymbol{\Delta})+o\left(\delta c_{u}\right) \\
& =\mathbf{f}+\mathbf{D}_{f, R}^{\mathrm{alg}}\left(\sum_{k=1}^{K} \delta a_{k}^{2}\left(\mathbf{e}\left(f_{k}\right) \otimes_{c} \mathbf{e}^{H}\left(f_{k}\right)\right)+\delta c_{u} \operatorname{Vec}\left(\mathbf{B} \mathbf{B}^{H}\right)\right)+o(\delta \boldsymbol{\Delta})+o\left(\delta c_{u}\right),
\end{aligned}
$$

where $\operatorname{Vec}\left(\mathbf{e}\left(f_{k}\right) \mathbf{e}^{H}\left(f_{k}\right)\right)=\mathbf{e}\left(f_{k}\right) \otimes_{c} \mathbf{e}^{H}\left(f_{k}\right)$ is used in the third equality. Therefore the following constraints upon $\mathbf{D}_{f, R}^{\text {alg hold: }}$

$$
\mathbf{D}_{f, R}^{\text {alg }}\left[\mathbf{e}\left(f_{k}\right) \otimes_{c} \mathbf{e}^{H}\left(f_{k}\right)\right]=\mathbf{0}, \quad k=1, \ldots, K \quad \text { and } \quad \mathbf{D}_{f, R}^{\mathrm{alg}} \operatorname{Vec}\left(\mathbf{B B}^{H}\right)=\mathbf{0}
$$


and using the expression (2.8), the proof follows.

\section{References}

[1] E.J. Hannan, Multiple time series, New York: Wiley, 1970.

[2] P.J. Brockwell, R.A. Davis, Time series, theory and methods, Second Edition, Springer Verlag, 1991.

[3] B. Porat, Digital processing of random signals, Theory and Methods, Prentice Hall, 1993.

[4] M. Rosenblatt, Stationary sequences and random fields, Birkhäuser, 1985.

[5] T.H. Li, B. Kedem and S. Yakowitz, "Asymptotic normality of sample autocovariances with an application in frequency estimation," Stochastic Processes and their Applications, vol. 52, pp. 329-349, 1994.

[6] B. Picinbono, "Second-order complex random vectors and normal distributions," IEEE Trans. Signal Processing, vol. 44, no. 10, pp. 2637-2640, Oct. 1996.

[7] F.D. Neeser and J.L. Massey, "Proper complex random processes with applications to information theory," IEEE Trans. Inform. Theory, vol.39, no. 4, pp. 1293-1302, July 1993.

[8] P. Billingsley, Probability and measure, Second Edition, John Wiley and Sons, 1986.

[9] A. Gorokhov, Y. Abramovich and J.F. Böhme, "Unified analysis of DOA estimation algorithms for covariance matrix transforms," Signal Processing, vol. 55, pp. 107-115, 1996.

[10] P. Stoica, T. Söderström and Funan Ti, "Overdetermined Yule-Walker estimation of the frequencies of multiple sinusoids: Accuracy aspects," Signal Processing, vol. 16, pp. 155-174, 1989.

[11] J.F. Cardoso, E. Moulines, "Asymptotic performance analysis of direction-finding algorithms based on fourthorder cumulants," IEEE Trans. Signal Processing, vol. 43, no. 1, pp. 214-224, Jan. 1995.

[12] R.J. Serfling, Approximation theorems of mathematical statistics, John Wileyand Sons, 1980.

[13] P. Stoica, T. Söderström, "Statistical analysis of MUSIC and subspace rotation estimates of sinusoidal frequencies," IEEE Trans. Signal Processing, vol. 39, no. 8, pp. 1836-1847, August 1991.

[14] G. Bienvenu, "Influence of the spatial coherence of the background noise on high resolution passive methods," in Proc. ICASSP, Washington, pp. 306-309, April 1979.

[15] S. Orfanidis, Optimum signal processing, an introduction, Macmillan, 1988. 
Biographie Jean Pierre Delmas was born in France in 1950. He received the engineering degree from Ecole Centrale de Lyon in 1973 and the Certificat d'études supérieures from the Ecole Nationale Supérieure des Télécommunications in 1982. Since 1980 he has been with the Institut National des Télécommunications where he is currently Professor. His teaching and research interests include Statistical Signal Processing. 